\title{
Primary Chemotherapy in a 47-Year-Old Patient with Giant Ulcerative and Necrotizing Nonseminomatous Testicular Germ Cell Tumor
}

\author{
Sophia Stock ${ }^{a} \quad J_{\text {Jian Marcon }}{ }^{b} \quad{\text { Michael Chaloupkab }{ }^{b} \text { Armin Becker }}^{b}$ \\ Wolfgang G. Kunz ${ }^{c}$ Steffen Ormanns ${ }^{d}$ Theresia Pichler ${ }^{\mathrm{C}}$ \\ Friederike H.A. Mumm ${ }^{a}$ e Julian W. Holch ${ }^{a, e, f}$ Lars H. Lindnera \\ aDepartment of Medicine III, University Hospital, LMU Munich, Munich, Germany; \\ ${ }^{b}$ Department of Urology, University Hospital, LMU Munich, Munich, Germany; 'Department \\ of Radiology, University Hospital, LMU Munich, Munich, Germany; Institute of Pathology, \\ Faculty of Medicine, LMU Munich, Munich, Germany; ${ }^{e}$ Comprehensive Cancer Center

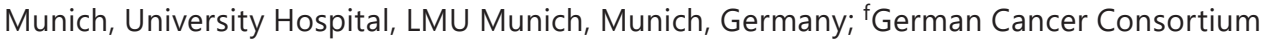 \\ (DKTK), Partner Site Munich and German Cancer Research Centre (DKFZ), Heidelberg, \\ Germany
}

\section{Keywords}

Cancer survivors · Germ cell tumor · Orchiectomy · Primary chemotherapy · Psychosocial distress

\begin{abstract}
Testicular cancer is a rare disease; however, cure rates are high for all tumor stages. Mostly, the disease is diagnosed in an early (local) stage. We report the case of a 47-year-old male patient with a giant nonseminomatous germ cell tumor. At the time of diagnosis, the patient demonstrated a necrotizing and ulcerating growing mass in the left scrotum with an approximate size of $22 \times 18 \mathrm{~cm}$. According to the prognostic classification of the International Germ Cell Cancer Collaborative Group (IGCCCG 1997), the patient exhibited a high-risk profile due to alpha-fetoprotein $>10,000 \mathrm{ng} / \mathrm{mL}$ and lactate dehydrogenase $>10 \times$ the upper limit of normal in serum. Primary orchiectomy was infeasible due to the tumor's size, the patient's poor general condition and initial intensive care unit treatment. Primary systemic chemotherapy was applied. After 3 cycles of cisplatin, etoposide and bleomycin, along with 1 cycle of cisplatin, etoposide and ifosfamide, tumor resection with histomorphological examination showed a complete pathological response. Despite the delayed initiation of the therapy, primary che-
\end{abstract}

J.W. Holch and L.H. Lindner contributed equally to this study. 
motherapy was completed timely and showed promising results. Reasons for the late hospitalization were personal responsibilities regarding his family. Better awareness and knowledge of testicular cancer among young men might prevent the here reported delay of medical consultation and avoid testicular tumors of such enormous size. Psychosocial assessment and distress management is important as an integral part of comprehensive care of testicular cancer patients.

(C) 2021 The Author(s).

Published by S. Karger AG, Basel

\section{Introduction}

Testicular cancer is a rare disease [1]. Notably, cure rates are $>90 \%$ for all stages due to consistent use of appropriate therapy concepts [1]. International recommendations and guidelines define the use of chemotherapy, surgery and radiation as well as the type and duration of its use depending on histology, tumor stage and risk factors [1]. Classification of germ cell tumors is performed by histological criteria and tumor markers [1]. Germ cell tumors are defined as seminomatous or nonseminomatous. Mixed tumors and cases with alpha-fetoprotein (AFP) elevation are considered as nonseminomatous. The prognostic classification of the International Germ Cell Cancer Collaborative Group (IGCCCG 1997) define three prognosis groups for nonseminomatous tumors by clinical and laboratory criteria [2]. Mostly, the disease is diagnosed at an early (local) stage [1]. Painless enlargement, swelling or circumscribed hardening of the testicles are early symptoms. Primary orchiectomy is the standard treatment. Depending on the stage, prognostic classification and further risk factors, patients with nonseminomatous tumors are managed with surveillance or treated with 1-4 cycles of chemotherapy after primary orchiectomy. Stage I nonseminomatous germ cell tumors with risk factors (e.g., vascular or lymphatic invasion) are usually treated with 1 cycle of cisplatin, etoposide and bleomycin (PEB). Cases with advanced-stage tumor and elevated tumor markers usually receive 3-4 cycles of PEB or cisplatin, etoposide and ifosfamide (PEI) depending on the prognosis group. Patients with initially advanced or acute life-threatening disease receive chemotherapy first and undergo delayed orchiectomy afterward [3].

We report the case of a 47-year-old male patient diagnosed with a giant nonseminomatous testicular tumor in the left scrotum and multiple lymph node metastases. Due to the tumor size and patient's poor general condition, he received 4 cycles of primary chemotherapy followed by tumor resection.

\section{Case Presentation}

A 47-year-old man was transferred to our university hospital in March 2020 because of an ulcerating testicular tumor. For about 3 years, the patient had noted a progredient testicular mass without consulting with a doctor. Some days before hospitalization, the patient noticed ulcerating scrotal wounds and fever. He had also noticed a swelling, redness and strong pain in the left leg for about 3 weeks. The patient had no preexisting conditions, long-term medication or history of infections or cancer.

Initial computed tomography (CT) scanning showed an $\sim 22 \times 18 \mathrm{~cm}$ ulcerating testicular mass with para-aortic, iliac and inguinal lymph node metastases on both sides (Fig. 1a-c). Moreover, an extensive thrombosis of the deep leg veins of the left upper and partially lower leg with an increase in circumference as well as subcutaneous and perifascial edema was detected. No parenchymatous distant metastases were found. Antibiotic treatment and anti- 
a
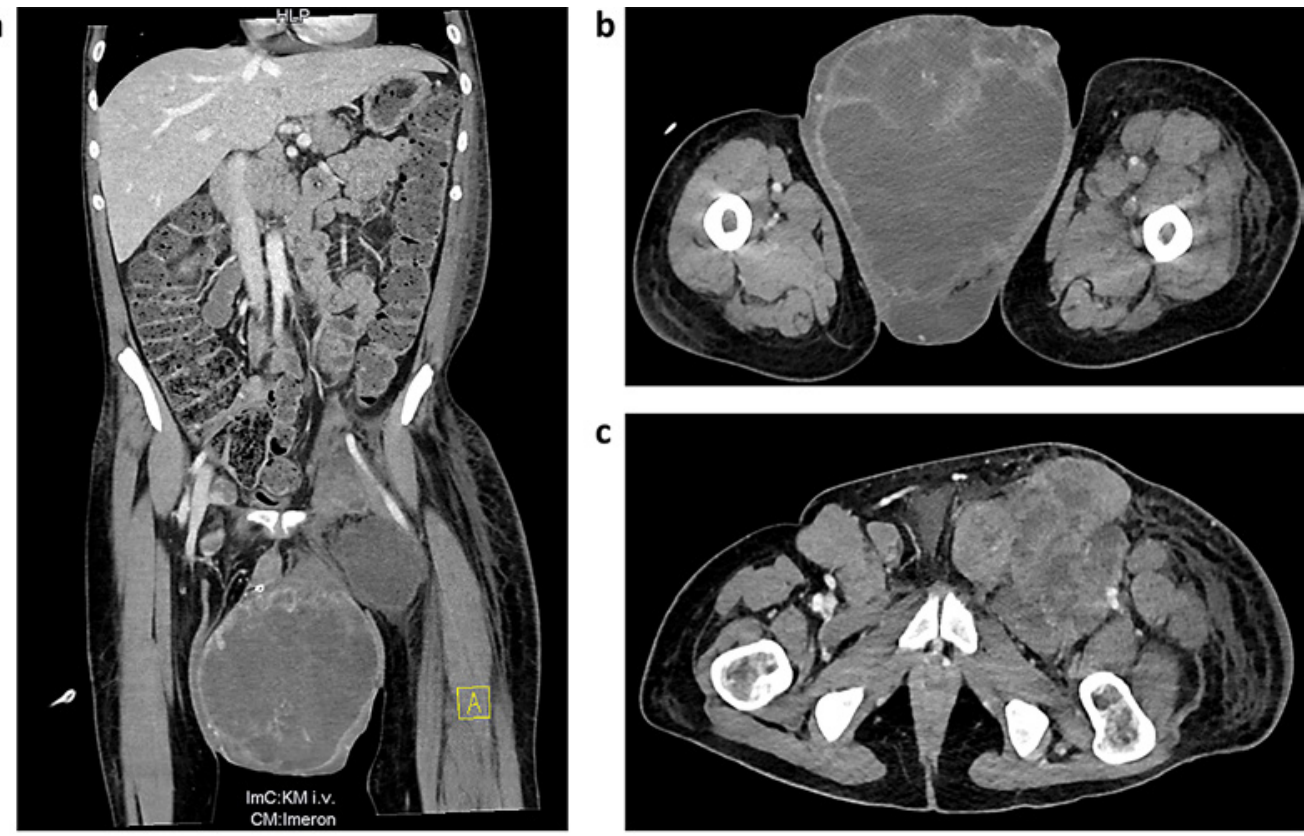

C

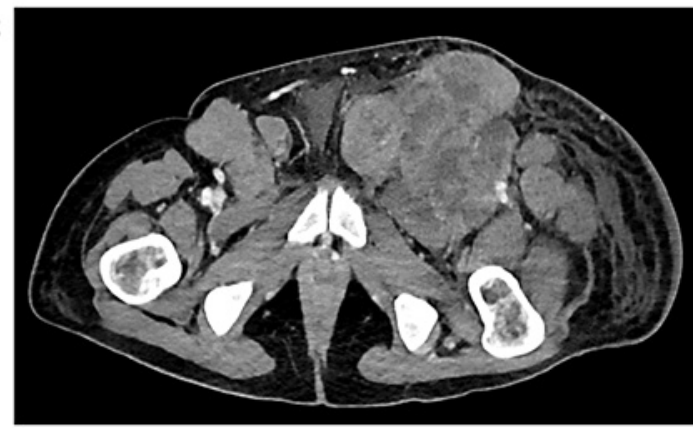

d

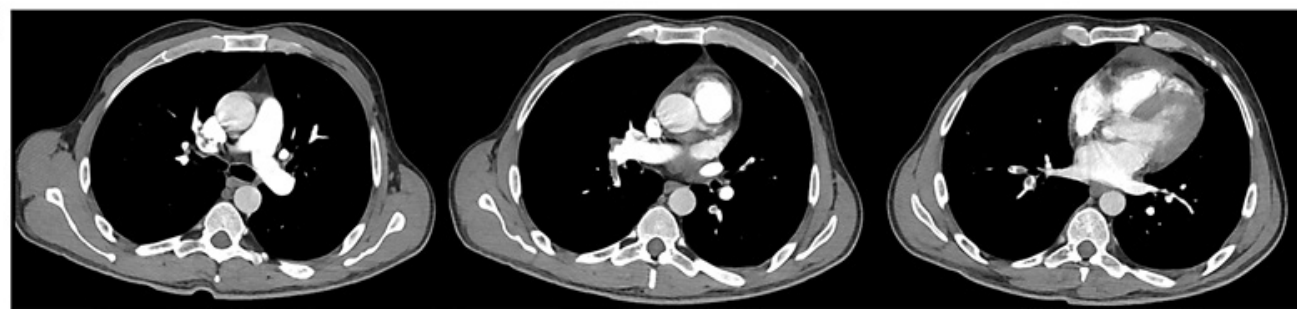

Fig. 1. Initial computed tomography (CT) scan showing the germ cell tumor in the coronal plane (a) and axial planes (b, c). CT angiography showing pulmonary artery embolization in the axial plane (d).

coagulant therapy with the low-molecular-weight heparin tinzaparin were initiated. On March 7, 2020, the patient developed a hemorrhagic shock due to tumor bleeding and was transferred to the intensive care unit (ICU). CT angiography showed bilateral acute pulmonary embolism with right heart strain and pulmonary infarction in the right middle lobe (Fig. 1d). Anticoagulation was switched to a heparin syringe pump despite the bleeding tumor as the pulmonary embolism severity index (PESI) was high with a 30 -day mortality $>10 \%$. After administration of volume and blood products, the patient stabilized and was transferred to the Department of Hematology and Oncology. Heparinization was later switched back to tinzaparin. Imaging was completed with magnetic resonance imaging of the head, showing no brain metastases. Additionally, bone metastases could be excluded by single-photon emission computed tomography.

Psycho-oncological support was offered to the patient. During the first consultation, the patient reported psychosocial distress. The predominant topics were the psychosocial circumstances preceding hospitalization - for example, having „no time for self-care “ because of financial responsibilities, caring for his family and a demanding job. Aspects of embarrassment were addressed by the patient. However, in view of the life-threatening situation, he was not willing to be more specific. It seemed that a more in-depth conversation about this emotion might have been too demanding. In this case, the patient benefited from the therapist's containment. After 2-3 consultations, he was willing to involve friends for further social 
Table 1. Time course of tumor marker and hormone levels in serum

\begin{tabular}{lcrrrrl}
\hline Date & $\begin{array}{l}\text { Beta-hCG, } \\
\mathrm{mIU} / \mathrm{mL}\end{array}$ & $\begin{array}{l}\text { AFP, } \\
\mathrm{ng} / \mathrm{mL}\end{array}$ & $\begin{array}{l}\text { LDH, } \\
\mathrm{U} / \mathrm{L}\end{array}$ & $\begin{array}{l}\text { LH, } \\
\mathrm{U} / \mathrm{L}\end{array}$ & $\begin{array}{l}\text { FSH, } \\
\text { U/L }\end{array}$ & $\begin{array}{l}\text { Testosterone, } \\
\mathrm{ng} / \mathrm{mL}\end{array}$ \\
\hline March 7, 2020 & 346 & - & 4,234 & - & - & - \\
March 9, 2020 & - & 38,378 & 2,868 & - & - & - \\
March 10, 2020 & - & - & 2,609 & $<0.3$ & $<0.3$ & 0.7 \\
March 20, 2020 & - & - & 1,220 & - & - & - \\
March 24, 2020 & - & - & 617 & - & - & - \\
April 8, 2020 & - & - & 317 & 3.0 & - & - \\
April 29, 2020 & - & - & 313 & - & - & 5.7 \\
May 5, 2020 & $<2$ & 465 & 160 & - & - & - \\
May 27, 2020 & - & 56.7 & 154 & - & - & - \\
June 4, 2020 & - & - & 90 & - & - & - \\
June 26, 2020 & $<2$ & 7.8 & 315 & - & - & - \\
July 6, 2020 & $<2$ & 5.1 & 179 & 22.0 & 31.4 & 5.9 \\
September 3, 2020 & $<2$ & 3.3 & 173 & - & - & - \\
September 11, 2020 & $<2$ & 3.3 & 237 & - & - & - \\
\hline
\end{tabular}

Beta-hCG, beta-human chorionic gonadotropin; AFP, alpha-fetoprotein; LDH, lactate dehydrogenase; LH, luteinizing hormone; FSH, follicle-stimulating hormone.

support. Notably, the patient showed high compliance during medical treatment, which may be interpreted as a kind of compensation for the delayed consultation.

Initial assessment of serum tumor markers revealed high levels of lactate dehydrogenase (LDH), beta-human chorionic gonadotropin and AFP (Table 1). Additional assessment of serum hormones revealed low levels of luteinizing hormone, follicle-stimulating hormone and testosterone, revealing a hypogonadotropic hypogonadism in which negative feedback may have been involved (Table 1). Due to the detected serum hormone levels, we considered a Leydig cell tumor as the most probable differential diagnosis.

Histomorphological investigation of a tumor sample obtained by inguinal lymph node biopsy predominantly showed scar-like fibrosclerosis and subtotally necrotic infiltrates of a small cell neoplasia. The morphology and immunophenotype were consistent with lymph node metastasis of a clinically suspected germ cell neoplasia, most likely a classic seminoma. Immunohistochemical stainings of the sparse vital tumor cells showed a distinct and strong expression of SALL4, podoplanin and OCT4, but no expression of PLAP or CD45. CAL1 was focally, though most probably aberrantly, expressed. CD30 expression was not detected. Although histopathology suggested a pure classic seminoma as the most probable differential diagnosis, the tumor was considered as nonseminoma due to a strongly increased AFP level $>10,000 \mathrm{ng} / \mathrm{mL}$.

According to the IGCCCG prognostic classification, the patient had a high-risk profile due to AFP being $>10,000 \mathrm{ng} / \mathrm{mL}$ and $\mathrm{LDH}>10 \times$ the upper limit of normal [2]. After an interdisciplinary evaluation, primary preoperative chemotherapy with 4 cycles of PEB was determined as the therapeutic approach. Initially, 3 cycles of cisplatin $\left(20 \mathrm{mg} / \mathrm{m}^{2}\right)$ on days $1-5$, etoposide $\left(100 \mathrm{mg} / \mathrm{m}^{2}\right)$ on days $1-5$, and bleomycin $(30 \mathrm{mg})$ on days 1,8 and 15 were performed with repetition after 21 days. Upon restaging after 1 cycle in mid-March 2020, with a decrease in size of the primary bulk $(\sim 20 \times 16 \mathrm{~cm}), 2$ more cycles of PEB were applied. Chemotherapy was well tolerated besides a temporary hearing impairment after the 2nd cycle. Audiometry in mid-April 2020 revealed an inner ear deterioration in the high frequency range on both sides of about $20 \mathrm{~dB}$ compared to a previous audiogram in March 2020 (CTCAE grade 1; CTCAE v5.0). Another control audiometry showed no further change of the hearing 

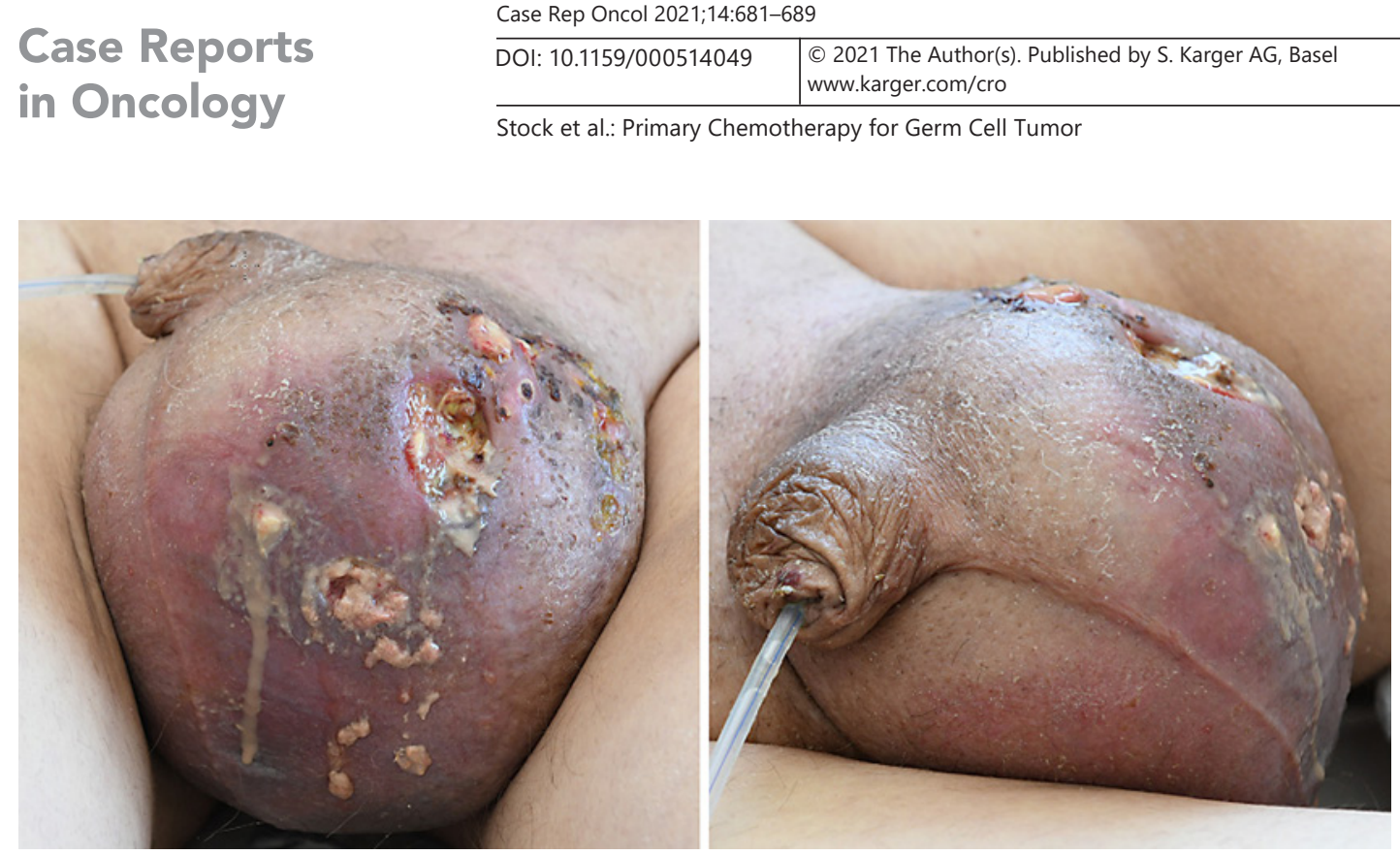

Fig. 2. Photographs of the ulcerative testicular mass in April 2020.

threshold. The patient reported a continuous improvement of his symptoms with no affection of his daily life. Due to chemotherapy-associated hematotoxicity, a blood transfusion was performed after the 3 rd cycle. Tumor marker monitoring revealed a significant reduction in LDH, beta-human chorionic gonadotropin and AFP (Table 1). A pulmonary function test before the 4th cycle showed a deterioration of the diffusion capacity of the lung for carbon monoxide (DLCO) with $41 \%$ of the predicted value. The patient was asymptomatic, and no intervention was indicated (CTCAE grade 1; CTCAE v5.0). As there was a contraindication for bleomycin, the 4 th cycle was performed without bleomycin as PEI with cisplatin $\left(20 \mathrm{mg} / \mathrm{m}^{2}\right)$, etoposide $\left(75 \mathrm{mg} / \mathrm{m}^{2}\right)$ and ifosfamide $\left(1,200 \mathrm{mg} / \mathrm{m}^{2}\right)$ each on days $1-5$. The 4 th cycle was well tolerated.

Throughout the systemic treatment, the patient received multiple surgical wound controls and local debridement. A macroscopically visible tumor decay and reduction without evidence of superinfection was observed (Fig. 2). The patient had multiple episodes of fever and elevated infection parameters in serum during his prolonged hospitalization. Antibiotic therapy was applied almost continuously according to the sensitivity of the microorganisms depending on the antibiogram. No ICU stay or surgery was necessary during the application of chemotherapy.

On June 10,2020, positron emission tomography and CT imaging after 4 cycles of chemotherapy showed a decreasing scrotal mass $(\sim 9.5 \times 9.5 \mathrm{~cm})$ with still existing contact to the penis root (Fig. 3). The lymph node metastases had shrunk significantly. No new hematogenic or lymphogenic lesions were detected. Furthermore, an entrapment of the left iliac vessels by a lymph node metastasis with consecutively unchanged thrombosis of the left external iliac vein was still present.

After a repeated interdisciplinary evaluation, the extent of tumor resection was defined. On July 7, 2020, radical inguinal orchidectomy, inguinal left-sided lymphadenectomy and partial resection of affected scrotal tissue were performed. It was possible to preserve the right testicle in its testicular layers. Furthermore, it was possible to separate the entire penis from the left-sided testicular tumor so that all compartments could be preserved. Macroscopic analysis of the removed tumor revealed a size of $\sim 10 \times 10 \times 6 \mathrm{~cm}$. Upon histomorphological examination of the resected specimen, no more vital tumor cells were found. The findings were well compatible with a testicular tumor with complete tumor regression after

\section{Karger's}



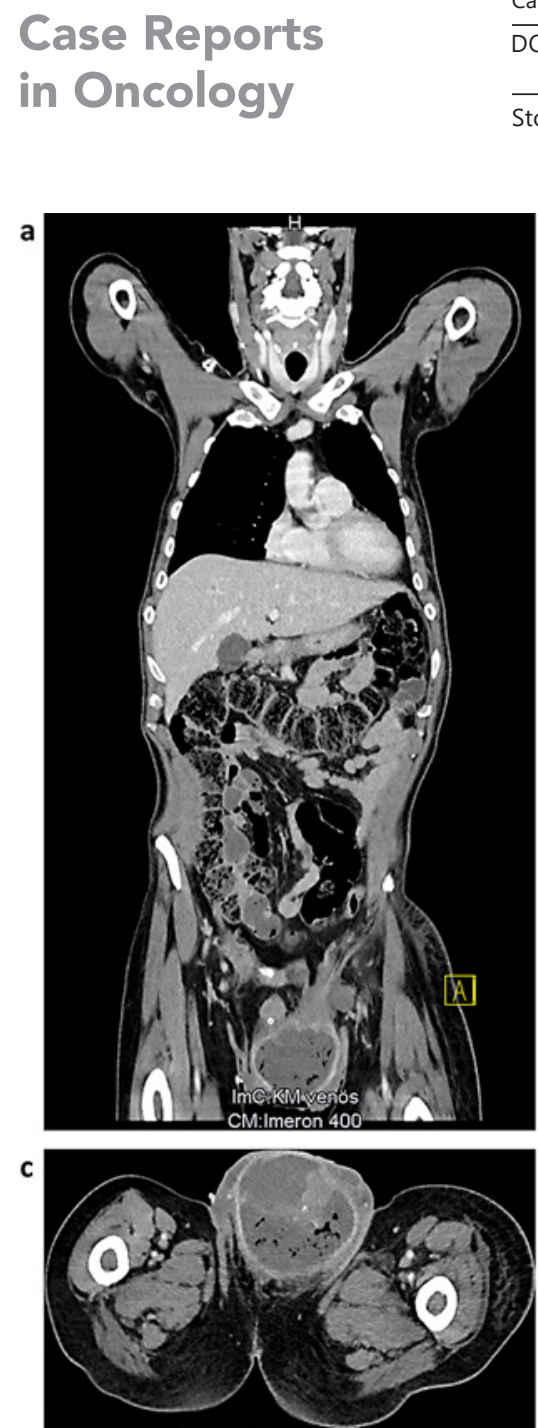

Fig. 3. Computed tomography scan showing the germ cell tumor in the coronal plane (a) and in the axial plane (c), as well as positron emission tomography-computed tomography showing the germ cell tumor in the coronal plane (b) and the axial plane (d), after 4 cycles of systemic chemotherapy in June 2020.

primary chemotherapy, so that no further statements about the entity and composition could be made. Furthermore, two analyzed inguinal lymph nodes showed a complete regression, since histomorphological examination detected totally necrotic tissue. Therefore, the response to preoperative treatment was classified as a complete pathological response. Postoperative tumor classification (UICC 8th edition 2017) was ypT0, ypN0 (0/2 lymph nodes), L0, V0, Pn0 with an R0 situation. A standardized follow-up schedule without further adjuvant treatment was determined and performed.

\section{Discussion}

Standard therapy of germ cell tumors is primary orchiectomy, followed by surveillance, radiotherapy or chemotherapy depending on the stage, histology and further risk factors. After orchiectomy, standard therapy for patients with nonseminomatous tumors in an advanced stage (stage $\geq \mathrm{II}$ ) is 3-4 cycles of PEB depending on risk classification. An alternative chemotherapy regime is PEI, having a similar effect with more myelotoxicity [4]. Recently, more intensive chemotherapy strategies have been investigated for patients with interme- 
diate and poor prognoses. If chemotherapy is intensified as a primary high-dose (HD) chemotherapy, 3-4 sequential cycles of HD-PEI plus stem cell support should be performed [5]. It was shown that patients with a poor prognosis had survival rates of $>70 \%$ when treated with HD-PEI as primary therapy [5]. General use of primary HD chemotherapy, however, is currently still not the standard. HD chemotherapy was not an option in this case due to a necrotic, superinfected and bleeding tumor bulk. For patients initially ineligible for surgery because of an advanced and acutely life-threatening disease, primary chemotherapy is the favored therapy [6, 7]. In this case, hospitalization occurred due to fever, thrombosis of deep leg veins, bilateral acute pulmonary embolism and tumor bleeding leading to ICU treatment. Because of the patient's poor general condition and acute life-threatening disease, primary orchiectomy was not an option. After 4 cycles of chemotherapy, orchiectomy and inguinal lymphadenectomy were performed. Notably, during chemotherapy, macroscopically visible necrosis was managed by local wound control and debridement without the need for surgical intervention (e.g., due to wound infection or bleeding).

Nowadays, cases with very large tumor sizes are rare. In most cases, patients notice early symptoms. Screening for testicular cancer is usually performed by self-examination or examination by an experienced physician. A general screening for early detection of testicular tumors in asymptomatic young males is not recommended, as the incidence is low and even patients with advanced cancer have favorable outcomes [8]. In case of a suspected testicular tumor, specific follow-up tests are required for diagnostic confirmation. Physicians should inform patients with clinical risk factors for testicular cancer (e.g., undescended testes, testicular intraepithelial neoplasia, history of testicular cancer and hereditary disposition). In this case, the patient had noticed symptoms about 3 years before he was finally hospitalized due to the life-threatening emergency. An analysis of 168 patients with nonseminomatous germ cell tumor revealed a median primary tumor size of $3.2 \mathrm{~cm}$ [9]. Cases with tumor sizes $>10 \mathrm{~cm}$ were rare [9]. Here, testicular cancer was diagnosed with a size of $\sim 22 \times 18 \mathrm{~cm}$ (measured by CT). Primary tumor size is associated with clinical stage, pathological stage and laterality [9].

Sociocultural norms as well as deficits of knowledge and awareness may lead to a lack of health-related knowledge, causing an unnecessary delay of medical consultation at an early stage [10]. It was reported that young men have little knowledge about their risk for testicular cancer, early testicular cancer signs and performance of a self-examination [10]. This highlights the need to increase awareness and education programs tailored to the target group, such as mass media campaigns. Psychological issues also play an important role for long-term survivors, especially as testicular cancer occurs at a young age. Here, mainly repression and embarrassment were the reasons leading to the late medical consultation. Perception of masculinity, sexual relationships and attractiveness plus fertility and sexual function is highly associated with the testes, especially by young men [11]. Sexuality-related concerns may lead to elevated distress levels (e.g., anxiety and depression), fear of the future and loss of control [12]. Patients in a long-term relationship mostly receive more social support, have a higher self-esteem and overall better mental health than singles [13]. However, therapy-associated sexuality and fertility problems may cause psychological stress to young couples with incomplete family planning [13]. Here, the patient was single and childless; however, sperm analysis and cryopreservation were declined. The quality of life of long-term survivors is influenced by individual health, sexual relationships and work problems [14]. Sociocultural differences in sexuality, masculinity and fertility have a direct impact on quality of life [14]. Therefore, psycho-oncological support should routinely address these issues during follow-up care, when patients may have more resources to talk about more complex issues and emotions such as embarrassment. Even more so because high levels of distress, mainly anxiety and fear of cancer recurrence, are reported for testicular cancer survivors [14, 15].

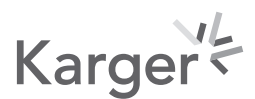




\section{Conclusion}

This case of a giant nonseminomatous testicular tumor successfully treated with primary chemotherapy without major complications is remarkable. Psychological aspects and sociocultural norms play an important role in testicular cancer discovery and throughout treatment. For improved management of these patients, psychologically supportive intervention and a biopsychosocial approach have to play a role.

\section{Acknowledgements}

We thank the patient described for allowing us to share details on his case.

\section{Statement of Ethics}

Patient anonymity and confidentiality of all information have been preserved. Written informed consent was obtained from the patient for publication of this case report and any accompanying images.

\section{Conflict of Interest Statement}

J.W.H. served on an advisory board for Roche and Pierre Fabre, and has received honoraria from Roche and travel support from Novartis.

\section{Funding Sources}

This research did not receive any specific grant from funding agencies in public, commercial, or not-for-profit sectors.

\section{Author Contributions}

All individuals who qualify as authors have been listed. S.S. was responsible for the design of the case report, for interpretation of the patient data and for drafting of the manuscript. S.S., J.W.H. and L.H.L. were responsible for oncological care of the patient. J.M., M.C. and A.B. were responsible for urological care of the patient. W.G.K. described the radiological data. S.O. was responsible for the pathology report. T.P. and F.H.A.M. were responsible for psychooncological support of the patient. J.W.H. and L.H.L. reviewed and critically edited the paper. All authors reviewed the manuscript draft. All authors read and approved the final version of the manuscript.

\section{Karger'k}




\section{Case Reports in Oncology}

\begin{tabular}{l|l}
\hline Case Rep Oncol 2021;14:681-689 \\
\hline DOI: 10.1159/000514049 & $\begin{array}{l}\text { ○ 2021 The Author(s). Published by S. Karger AG, Basel } \\
\text { www.karger.com/cro }\end{array}$ \\
\hline
\end{tabular}

Stock et al.: Primary Chemotherapy for Germ Cell Tumor

\section{References}

1 Albers P, Albrecht W, Algaba F, Bokemeyer C, Cohn-Cedermark G, Fizazi K, et al. Guidelines on testicular cancer: 2015 update. Eur Urol. 2015 Dec;68(6):1054-68.

2 International Germ Cell Consensus Classification: a prognostic factor-based staging system for metastatic germ cell cancers. International Germ Cell Cancer Collaborative Group. J Clin Oncol. 1997 Feb;15(2):594-603.

3 Beyer J, Albers P, Altena R, Aparicio J, Bokemeyer C, Busch J, et al. Maintaining success, reducing treatment burden, focusing on survivorship: highlights from the third European Consensus Conference on Diagnosis and Treatment of Germ-Cell Cancer. Ann Oncol. 2013 Apr;24(4):878-88.

4 de Wit R, Stoter G, Sleijfer DT, Neijt JP, ten Bokkel Huinink WW, de Prijck L, et al. Four cycles of BEP vs four cycles of VIP in patients with intermediate-prognosis metastatic testicular non-seminoma: a randomized study of the EORTC Genitourinary Tract Cancer Cooperative Group. European Organization for Research and Treatment of Cancer. Br J Cancer. 1998 Sep;78(6):828-32.

5 Schmoll HJ, Kollmannsberger C, Metzner B, Hartmann JT, Schleucher N, Schöffski P, et al. Long-term results of first-line sequential high-dose etoposide, ifosfamide, and cisplatin chemotherapy plus autologous stem cell support for patients with advanced metastatic germ cell cancer: an extended phase I/II study of the German Testicular Cancer Study Group. J Clin Oncol. 2003 Nov 15;21(22):4083-91.

6 Ondrus D, Hornák M, Breza J, Mat'oska J, Schnorrer M, Belan V, et al. Delayed orchiectomy after chemotherapy in patients with advanced testicular cancer. Int Urol Nephrol. 2001;32(4):665-7.

7 Ramani VA, Grey BR, Addla SK, Dunham MP, Sangar VK, Clarke NW. Histological outcome of delayed orchidectomy after primary chemotherapy for metastatic germ cell tumour of the testis. Clin Oncol (R Coll Radiol). 2008 Apr;20(3):247-52.

8 Ilic D, Misso ML. Screening for testicular cancer. Cochrane Database Syst Rev. 2011 Feb;16(2):CD007853.

9 Dieckmann KP, Richter-Simonsen H, Kulejewski M, Ikogho R, Zecha H, Anheuser P, et al. Testicular germ-cell tumours: a descriptive analysis of clinical characteristics at first presentation. Urol Int. 2018;100(4):409-19.

10 Ugboma HA, Aburoma HL. Public awareness of testicular cancer and testicular self-examination in academic environments: a lost opportunity. Clinics (Sao Paulo). 2011;66(7):1125-8.

11 Carpentier MY, Fortenberry JD. Romantic and sexual relationships, body image, and fertility in adolescent and young adult testicular cancer survivors: a review of the literature. J Adolesc Health. 2010 Aug;47(2):115-25.

12 Siafaka V, Hyphantis TN, Alamanos I, Fountzilas G, Skarlos D, Pectasides D, et al. Personality factors associated with psychological distress in testicular cancer survivors. J Pers Assess. 2008 Jul; 90(4):348-55.

13 Condello C, Rescigno P, Ottaviano M, Nappi L, Tortora M, de Placido S, et al. Clinical features and psychological aspects of the decision-making process in stage I testicular germ cell tumors. Future Oncol. 2018 Jul;14(16): 1591-9.

14 Schepisi G, De Padova S, De Lisi D, Casadei C, Meggiolaro E, Ruffilli F, et al. Psychosocial issues in long-term survivors of testicular cancer. Front Endocrinol. 2019;10:113.

15 Kreiberg M, Bandak M, Lauritsen J, Andersen KK, Skøtt JW, Johansen C, et al. Psychological stress in long-term testicular cancer survivors: a Danish nationwide cohort study. J Cancer Surviv. 2020 Feb;14(1):72-9. 